\title{
Hydrogen Exchange and Covalent Modification: Focus on Biomolecular Structure, Dynamics, and Function. 18th Sanibel Conference on Mass Spectrometry
}

The spectacular successes of proteomics and bioinformatics in the post-genomic era resulted in an explosive growth of information on the make-up of complex networks of proteins interacting at the cellular level and beyond. Coupled with the avalanche of new highresolution protein structures, these advances raise hopes that rational manipulation of these networks aimed at achieving a desired therapeutic outcome will soon become possible. A simple inventory of interacting proteins and even the availability of their static structures, however, are often insufficient for understanding how the components of sophisticated biological machinery work together. It is now clear that the multiplicity of protein states, as well as transitions among them, are critical determinants of protein function as diverse as recognition, transport, and enzyme catalysis, to name a few.

Mass spectrometry now plays a visible role in protein characterization. Despite being a relative newcomer in analysis of higher order structure and dynamics of biopolymers, it is evolving as a powerful analytical tool capable of revealing intimate details of protein behavior. A variety of mass spectrometry-based experimental strategies aiming at characterizing both static and dynamic aspects of protein higher order structure were the focus of the 18th Sanibel Conference on Mass Spectrometry held at the Sundial Conference Center on Sanibel Island, Florida, in January 2006. The conference "Hydrogen Exchange and Covalent Modification: Focus on Biomolecular Structure, Dynamics and Function" brought together nearly 100 scientists who work in this area.

Several articles contributed by the Conference presenters for this Focus Issue of JASMS provide a snapshot of the current status of this field. The opening article by Walter Englander (University of Pennsylvania), one of the pioneers of protein hydrogen/deuterium exchange (HDX), provides a valuable historical background and places mass spectrometry in the context of other analytical techniques used in tandem with HDX. It is perhaps symbolic that the Conference, with its emphasis on deuterium as a structural probe, took place in the year that marks the 75th anniversary of deuterium discovery. Some milestones in adopting deuterium as a probe of protein

Published online September 8, 2006 structure and dynamics are shown on the cover of this issue, including Linderstrøm's famous gradient tube (bottom left corner, Hvidt and LinderstrømLang, Biochim. Biophys. Acta 1954, 14, 574) and the first instrument that monitored HDX by measuring mass change of the protein sample exposed to $\mathrm{D}_{2} \mathrm{O}$ vapor (top right corner, Burley et al. J. Text. Inst. 1955, $46, \mathrm{~T} 427)$. Although deuterium has been used as a probe for several decades (long before mass spectrometry entered this field), Englander reminds us (by highlighting some controversial issues with long histories) that interpretation of protein HDX measurements is not always straightforward. One such issue is explored in depth by Komives and her coworkers at the University of California, San Diego, who evaluate the correlation between solvent accessibility and HDX kinetics for globular and nonglobular proteins.

Six sequel articles in the Focus deal with various applications of protein HDX and mass spectrometry, ranging from mechanistic studies and quantitation of protein interaction to new methods of extracting information on protein dynamics from experimental data. Engen and coworkers (University of New Mexico) describe, using peak width measurements from HDX spectra, a means to characterize exchange kinetics. Deinzer and colleagues (Oregon State University) found, using HDX and pepsin digestion, that when some agonists bind to dimeric retinoic acid $\mathrm{X}$ receptor $\alpha$, slower deuterium uptake occurs compared with when the natural ligand, 9-cis-retinoic acid, binds. The binding of other agonists leads to poorer protection, and faster deuterium uptake.

Wysocki and coworkers (University of Arizona) provide an excellent example of solution HDX in their analysis of the dynamic properties of cytochrome $c 2$. Chitta, Gross, and their coworkers at the Washington University in St. Louis present a novel approach to obtain thermodynamic characteristics of polypeptide aggregation based on HDX measurements. A contribution from Dai and Fitzgerald (Duke University) focuses on defining the limitations of HDX MS-based thermodynamic measurements. Abzalimov and Kaltashov (University of Massachusetts at Amherst) describe a deconvolution procedure that allows local backbone dynamics to be evaluated based on the measurements 
that combine HDX in solution and protein ion fragmentation in the gas phase.

Three additional articles in the Focus cover covalent labeling. Vachet and his colleagues from the University of Massachusetts at Amherst illustrate how metal-catalyzed oxidation identifies the amino acids that interact with metals in metalloproteins. Tomer and coworkers (National Institute of Environmental Health Sciences) describe how chemical modification of surface lysine side chains, along with limited proteolysis, delineate the interaction surfaces of protein complexes. Lastly, Fabris and his coworkers at the University of Maryland Baltimore County explain how cross-linking agents can reveal the topology of folded structures of nucleic acids.
Taken together, contributions in this Focus Issue highlight the enormous capabilities of mass spectrometry in biophysics and structural biology. It is our hope that this issue will not only provide valuable reference material for those wishing to use mass spectrometry to investigate biopolymer architecture and dynamics, but also catalyze further exciting developments in these fields.

Igor A. Kaltashov, Program Chair 18th Sanibel Conference

John R. Engen, Program Chair 18th Sanibel Conference

Michael L. Gross, Editor-in-Chief

\section{(Cover design by Igor Kaltashov)}

